\title{
Fluchtpunkt Hellespont? - Einige Bemerkungen zur Renaissance frühbronzezeitlicher Keramiktraditionen in der anatolischen Eisenzeit ${ }^{1}$
}

Ein Schwerpunkt archäologischer und philologischer Forschungstätigkeit in Vorderasien liegt in dem Bestreben, das kulturelle Gepräge Kleinasiens und seiner angrenzenden Regionen nach dem endgültigen Zusammenbruch des hethitischen Großreiches gegen Ende des zweiten Jahrtausends v. Chr. für alle Gebiete gleichmäßig austariert zu interpretieren. ${ }^{2}$ Zwar stehen uns eine Menge an Erkenntnissen zur kritischen Phase der altorientalischen Großreiche gegen 1200 v. Chr. zur Verfügung, die durch eine oft unglückliche Verquickung von ökologischen und politischen Faktoren bereits im Vorfeld des Zusammenbruchs empfindlich geschwächt scheinen. ${ }^{3}$ Quellensituation und Chronologie sind in den Nachbargebieten ist aber oft zu unausgewogen bzw. unsicher, als daß die erheblichen sozialen und ökonomischen Umwälzungen in ihrer vollen Konsequenz für die weitere geschichtliche Entwicklung des Nahen Ostens schlüssig beschrieben und gedeutet werden könnten. ${ }^{4}$

Der reichen wissenschaftlichen Ernte aus den späten Zentren der orientalischen Großmächte, die zur Erhellung der Geschehnisse an der Schwelle des 1. vorchristlichen Jahrtausends unabdingbar sind (supra), steht eine weitgehende Unklarheit über das Schicksal und die weitere Entwicklung für das Umland der jeweiligen Zentralorte gegenüber - ein Forschungsdesiderat, daß erst in jüngerer Zeit verstärkt in Angriff genommen wurde. ${ }^{5}$

${ }^{1}$ Das Manuskript wurde im Februar 2006 abgeschlossen. Ich danke den Herren Rainer Czichon, Berlin und Oğuz Soysal, Chicago für ihre wertvollen Kommentare und Ratschläge bzgl. der Erstfassung des vorliegenden Textes. Besonderer Dank ergeht zudem an Herrn Hikmet Denizli, Direktor des Museums für Anatolische Zivilisationen in Ankara, für die Erteilung der Kalınkaya-Publikationsgenehmigung, sowie an Herrn Ben Claasz Coockson, Bilkent Universität für die Anfertigung der Zeichnungen.

2 Für aktuelle Diskussionsbeiträge zur kritischen Phase des Übergangs von der Spätbronze- zur Eisenzeit im mittelmeerischen und vorderorientalischen Raum vgl. Ward/Joukowsky (1992); Gitin u.a. (1998); Silberman (1998); Niemeier (1998); Fischer u. a (2003); Strobel (2005) mit weiterführender Lit.

3 Für eine ausführliche Darstellung siehe Klengel (1999), $309 \mathrm{ff}$. sowie für Literatur bis 1998 407f.; bes. Hoffner (1992), 50f.; Muscarella 1995, 91 ff.; Sürenhagen (1996), 286ff.; Seeher (2001), 623 ff. mit Lit. Zu Engpässen in der existenzsichernden Getreideversorgung vgl. speziell Klengel (1974), $170 \mathrm{ff}$.

4 Vgl. Bertram (2003), 250.

${ }^{5}$ Siehe u.a. dazu Ökse (1999), bes. 327 ff.; 359ff.; Tab. 5; Czichon (1997); Czichon (1998); Czichon (1999). 
Dank neu gewonnener Erkenntnisse zu stilistischen Eigenheiten früheisenzeitlicher Keramik läßt sich dem komplexen kulturellen Bild Vorderasiens im spätesten zweiten und frühen ersten Jahrtausend v. Chr. nun ein wichtiges Puzzlestück hinzufügen. Hermann Genz konnte jüngst in mehreren Beiträgen anschaulich darlegen, daß früh- und mitteleisenzeitliche Keramikformen, insbesondere bemalte Waren, ein sichtbares Erbe der frühen und mittleren anatolischen Bronzezeit mit sich tragen. ${ }^{6}$ So weisen z. B. Kannenformen aus den eisenzeitlichen Schichten des Büyükkaya von Boğazköy oder Eskiyapar deutliche Parallelen zu frühbronzezeitlichen Gefäßen aus zentralanatolischen Siedungen wie Alaca Höyük bzw. Nallıhan auf. ${ }^{7}$ Auch mittelbronzezeitliche Typen in Form von Krügen mit Kleeblattausguß, wie sie beispielsweise Nekropole Demircihöyük-Sarıket vorkommen, lassen sich stilistisch mit wesentlich jüngeren Gefäßen aus dem frühen ersten Jahrtausend v. Chr. in Verbindung bringen. ${ }^{8}$ Unabhängig davon verwies kürzlich Nicholas Postgate auf ähnliche Beobachtungen bei der Auswertung der Keramik des im Göksu-Tal bei Silifke gelegenen Kilise Tepe: auch hier tauchen in bronzezeitlicher Tradition dekorierte, bemalte Keramikprodukte plötzlich wieder in eisenzeitlichem Schichtzusammenhang auf. ${ }^{9}$

Dieses hier nur kurz skizzierte Phänomen erschöpft sich nun nicht in den bereits vorgestellten Keramiken, sondern erstreckt sich auf weitere, in diesem Fall jedoch vorwiegend im Nordwesten Kleinasiens beheimatete früheisenzeitliche Gefäßtypen: unser Augenmerk soll sich daher im Folgenden auf sogenannte „Buckelkeramik“ (vgl. Abb. 1,1; 3,1) richten, die v. a. aus den eisenzeitlichen Schichten Troias bekannt ist. Hier sind die charakteristisch mit einer Kombination aus alternierenden Riefen und Knubben (den namengebenden „Buckeln“) verzierten Gefäße Leitformen der Stufe Troia VIIb2. ${ }^{10}$ Die Keramik ist ohne Ausnahme handgefertigt, an Formen dominieren offene, z. T. bikonische, hauptsächlich aber S-förmige Wirtschaftsgefäße sowie ein- und zweihenklige Becher und Tassen mit z. T. überrandständigen Ellenbogenhenkeln. ${ }^{11}$ Die Gefäßoberflächen sind zumeist dunkel bis schwarz poliert. Der weiche, nur schwach bei niedrigen Temperaturen gebrannte Ton ist weniger grob gemagert als die zeitlich etwas früher einsetzende, aber ebenso fremd wirkende „Barbarischen Ware“. 12

Sowohl Herstellungstechnik als auch Formen und Verzierungsweisen sowohl von „Buckelkeramik“ als auch der „Barbarian Ware“ kennen keine Vorläufer in den älteren Schichten der Ansiedlung, so daß diese so andersartigen groben Waren traditionell mit der Neuankunft von thrakisch-südosteuropäischen Bevölkerungselementen in Verbindung gebracht werden, die in den Wirren der letzten Jahrzehnte des 2. Jahrtausends v. Chr. nach Anatolien gelangt sein sollen.

Demzufolge kämen als mögliche Vorläufer für die bereits ab Troia VIIa auftretende Barbarische Ware in erster Linie Keramikerzeugnisse der am unteren Donaulauf in Ost-

${ }^{6}$ Genz (2003), 187; Genz (2005).

7 Genz (2005), 77 Abb. 1.

8 Genz (2005), 79 Abb. 2 .

9 Postgate (2005), $27 \mathrm{f}$.

10 Blegen u. a. (1958), 165; Koppenhöfer (1997), 316; 346.

11 Koppenhöfer (2002), 679; 681f. Abb. 2-3.

12 Koppenhöfer (2002), 679. 
bulgarien beheimateten Colsogeni-Kultur sowie benachbarter Steppenkulturen in Frage. ${ }^{13}$ Die Ahnenreihe der Buckelkeramik würde von den ebenfalls westpontischen Kultur des Babadag letztendlich zu den Produkten der spätbronzezeitlichen mitteleuropäischen Urnenfelderkultur und ihrer späten Derivate wie der Pşeničevo-Gruppe reichen. ${ }^{14}$

Diese immer wieder zitierten Parallelen in Form von spätbronzezeitlicher Keramik aus dem Balkangebiet bzw. Südosteuropa ${ }^{15}$, die zur Stützung der balkanischen Migrationsthese ins Feld geführt werden, vermögen jedoch nicht vollends zu überzeugen: Die einschlägige Keramik der Siedlung Kastanas in Mazedonien besitzt zwar Ziermuster, die in ähnlicher Ausprägung auf troianischer Buckelkeramik beobachtet werden konnten. ${ }^{16}$ Die Gefäßformen lassen hingegen keine Vergleiche zu. ${ }^{17}$ Wirklich gute technische und formale Analogien zur „Knobbed Ware“ oder Buckelkeramik findet noch vereinzelt in dem keramischen Inventar des bereits zitierten Babadağ ${ }^{18}$, besseres Vergleichsmaterial für unsere Buckelkeramik aus Troia VIIb2 reduziert sich aber strenggenommen auf früheisenzeitliche Grabkammern von Dolmenanlagen in Türkisch-Thrakien ${ }^{19}$ sowie einzelne weitere Fundplätze in dieser Region. ${ }^{20}$

Weiterhin ist eine balkanische Überprägung (Nord)west- und Inneranatoliens in der frühen Eisenzeit kaum zu belegen. ${ }^{21}$ Lediglich einige grobe handgemachte, „balkanisch“ anmutende Gefäßscherben aus Gordion ${ }^{22}$ und evtl. aus Kaman-Kalehöyük ${ }^{23}$ im Landesinneren, die ähnlich wie in Troia keine Anknüpfungspunkte an ältere Keramiktechnologien $\mathrm{zu}$ besitzen scheinen, mögen von einem schwachen südosteuropäischen Einfluß zeugen.

Ein Blick nach Zentralanatolien lehrt jedoch, daß beinahe identisch geformte und verzierte Knubbentassen in Siedlungsschichten der dortigen späten Frühbronzezeit vorkommen. Ebenso wie ihre eisenzeitlichen Pendants besitzen sie neben charakteristischen, meist auf dem Bauchumbruch platzierten Knubben einen Dekor aus diagonal alternierenden, am Hals oft doppelt umlaufenden Kannelurengruppen, die beide als Zierat an frühbronzezeitlichen Metallgefäßen geläufig sind und auch von dort übernommen worden sein dürften. ${ }^{24}$ Aber nicht nur typische Ziermuster, auch Waren ${ }^{25}$ und Gefäßformen selbst

${ }^{13}$ Koppenhöfer (2002), 687 ff.; 703; Pinążek-Sikora (2002), 705 f.

14 Bereits vermutet von Bouzek (1985), 185 ff., der die handgemachten Waren mit den Keramiktraditionen der Urnenfelderzeit in Mitteleuropa sowie der Kulturstufe der subappeninischen Facies des PräProtovillanova in Beziehung setzt. Auch südserbische und westpontische Provinzen der Spätbronzezeit werden in diese Zusammenstellung mit einbezogen (ebd. 187); Koppenhöfer (2002), 703; Pinążek-Sikora (2002), $705 \mathrm{f}$.

15 Vgl. Hnila/Pieniazek-Sikora (2002), 97 f.; Becks (2003), 49.

16 Hochstätter (1984), 373ff.; Koppenhöfer (1997), 340.

17 Koppenhöfer (1997), 340.

${ }^{18}$ Koppenhöfer (1997), 337 ff.; 339 Abb. 26, 1-5.

19 Akman (1998), $67 \mathrm{ff} . ; 70$ Abb. 5.

20 Vgl. Özdoğan (1987), 14 f.; 31 ff. mit Abb.; Özdoğan (1993), 160 ff., bes. 162.

21 Genz (2000), 40.

${ }^{22}$ Sams (1992), 58f.; Abb. 8,1; Koppenhöfer (1997), 341; Voigt/Henrickson (2000), 342 ff.; bes. 355.

23 Omura (1991), 323; 332 Abb. 8,1.

24 Vgl. beispielsweise Toker/Öztürk (1992), 49 Abb. 23; 188 Nr. 23 (aus Grab H, mit 6 durchlochten Karneolperlen unterhalb Umbruch, durch Goldnägel gehalten) (Knubben); 50 Nr. 24; 129 Nr. 24 (aus 
zeigen zumindest bei kleineren, einhenkligen Tassenformen erstaunliche Übereinstimmungen: So findet sich die gedrungen breit-bikonische Form mit weitmundig-schrägem Ausguß ebenso im keramischen Fundus von Troia VIIb2 wieder. Angeführt werden könnten neben dem Exemplâr aus Karaoğlan (Abb. 1,2) weitere Stücke aus Ahlatlıbel ${ }^{26}$ und Koçumbeli ${ }^{27}$, die in Form und Verzierung auf bestechende Weise den eisenzeitlichen Buckeltassen aus Troia VIIb2 ähneln. Auch Alaca Höyük ${ }^{28}$ sowie der benachbarte Toptaştepe bei Kalınkaya können in der Frühen Bronzezeit mit knubben- und riefenbündelverzierten Keramiken aufwarten (vgl. Abb. 2; 3,2). Datiert werden können diese Gefäße in die entwickelte bzw. späte Frühbronzezeit (FBZ II/III nach anatolischer Terminologie), sprich der Mitte bis dem letzten Drittel des dritten vorchristlichen Jahrtausends. Dieser Befund läßt sich zeitlich hervorragend mit den bereits diskutierten bemalten Waren korrelieren, die über den eingangs geschilderten, durchaus überzeugend dargebrachten Analogieschluß als stilistische Vorläufer eisenzeitlicher Keramik aus Inneranatolien verstanden werden können. Da man unter diesen Voraussetzungen durch einen wenn auch schlichten formalen Vergleich zwischen frühbronzezeitlicher und eisenzeitlicher Buckelkeramik zu dem gleichen Ergebnis gelangen muß, liegt auch hier die Vermutung nahe, das ähnliche Faktoren eine „Wiedergeburt“ altanatolischer Stiltraditionen herbeiführten. Die von Nicholas Postgate und auch Hermann Genz vertretene Hypothese, das bestimmte Traditionen im Verlauf des zweiten Jahrtausend v. Chr. „in den Untergrund gingen“, um nach dem Zusammenbruch einer zentralen, kulturell überprägenden Ordnungsmacht wie des hethitischen Reiches wieder reanimiert zu werden ${ }^{29}$, würde dann als Erklärungsmodell ebenso für unsere Buckelkeramik greifen. Archäologisch Faßbares wie Zierstile und Fertigungstechniken hätten dann in den Randgebieten oder in kleinen dörflichen Gemeinschaften, die nicht von den kulturellen Synergieeffekten des Hethiterreiches erfaßt wurden, still überlebt, um im spätesten zweiten und frühesten ersten Jahrtausend v. Chr. wieder in unseren Gesichtskreis zu treten.

Die zu erwartenden Bevölkerungsverschiebungen auch innerhalb Anatoliens, die nach dem endbronzezeitlichen Kollaps der mittelmeerischen und nahöstlichen Großmächte gewiß unter tragischen Begleitumständen wie Hunger, Vertreibung und großer materieller

Grab K); ebenso Tolstikov/Treister 1996, 31, 3 (silberne Flasche aus Schatzfund „A“, Troia“) 36, 7 (goldener Becher aus gleichem Kontext) (Riefendekor).

25 Ein erheblicher Teil FBZ-III-zeitlicher Keramiken besitzt einen dickem schwarzen, hochpoliertem Überzug, der neben den bereits erwähnten Riefen und Knubben wohl zusätzlich einen „metallischen" Effekt erzeugen sollte. Siedlung und Gräberfeld von Kalınkaya erbrachten beispielsweise eine Vielzahl von dünnwandigen, bikonischen Formen mit ebendiesen Attributen. Ein erster Vorbericht ist für dieses Jahr vorgesehen.

26 Orthmann (1963), 24 ff.; Taf. 25, 5/55.58-60.

27 Anpubliziert bei Tezcan (1966), Taf. 13 oben; eine größere Anzahl riefen- und knubbenverzierter Tassen bzw. Tüllengefäße befinden sich in der Dauerausstellung des Museums der Middle East Technical University in Ankara, sind aber bislang nicht veröffentlicht.

28 Orthmann (1963), 32ff.; Taf. 45, 11/61-65. - hinzufügen ließe sich eventuell noch die mit groben breiten Riefen und Knubben dekorierte Keramik des am Schwarzen Meer gelegenen Dündartepe (Orthmann (1963), 45f.; Taf. 66, 16/25.26) - weitere gute Vergleichsstücke befinden sich in der Dauerausstellung der Museums für Anatolische Zivilisationen in Ankara und harren ebenfalls noch ihrer adäquaten Veröffentlichung.

${ }^{29}$ Vgl. Genz (2005); Postgate (2005), 27. 
Not vonstatten gingen, lassen sich in ihrem geographischen und sozialen Ausmaß bislang nur erahnen. ${ }^{30}$ Eine balkanische Zuwanderung nach Troia soll und kann jedenfalls, um dies klarzustellen, dadurch nicht ausgeschlossen werden. ${ }^{31}$ Nach der hier vorgenommen kritischen Neuinterpretation der sogenannten Buckelkeramik sollte man jedoch ebenso in Erwägung ziehen, daß die Neuankömmlinge aus Südosteuropa in der ehemals stolzen Feste am Hellespont auf entwurzelte Flüchtlingsfamilien aus Inneranatolien trafen.

\section{Literatur}

Alkım, U. B., Anatolien I, Genf 1968.

Becks, R., Troia VII: the Transition from the Late Bronze Age to the Early Iron Age, in: Fischer u. a. (2003), 41-53.

Bertram, J.-K., Tradition und Wandel im späten 2./frühen 1. Jahrtausend v. Chr. im Südkaukasusgebiet, in: Fischer u. a. (2003), 245-253.

Blegen, C. W. - Boulter, C. G. - Caskey, J. L. - Rawson, M., Troy IV. Settlements VIIa, VIIb and VIII, Princeton 1958

Bouzek, J., The Aegean, Anatolia and Europe: Cultural Interrelations in the Second Millennium B.C., Göteborg/Prag 1985.

Czichon, R. M., Studien zur Regionalgeschichte von Hattuša/Boğazköy 1996, MDOG 129 [1997] 89-102.

Czichon, R. M., Studien zur Regionalgeschichte von Hattuša/Boğazköy 1997, MDOG 130 [1998] 83-92.

Czichon, R. M., Studien zur Regionalgeschichte von Hattuša/Boğazköy 1998, MDOG 131 [1999] 47-56.

Fischer, B. - Genz, H. - Jean, E. - Köroğlu, K. (Hrsg.), Identifying Changes: The Transition from Bronze to Iron Ages in Anatolia and its Neighboring Regions. Proceedings of the International Workshop Istanbul, November 8-9, 2002, Istanbul 2003.

Genz, H., Die Eisenzeit in Zentralanatolien im Lichte der keramischen Funde vom Büyükkaya in Boğazköy/Hattuša. TÜBA-AR (Turkish Academy of Sciences Journal of Archaeology) 3 [2000] $35-54$.

Genz, H., The Early Iron Age in Central Anatolia, in: Fischer u. a. (2003), 179-191.

Genz, H., Thoughts on the origin of the Iron Age pottery traditions in central Anatolia, in: Çilingiroğlu, A. - Darbyshire, G. (Hrsg.), Anatolian Iron Ages 5. Proceedings of the Fifth Anatolian Iron Ages Colloquium held at Van, 6-10 August 2001, Ankara/London 2005, 75-84.

Gitin, S. - Mazar, A. - Stern, E. (Hrsg.), Mediterranean Peoples in Transition. Thirteenth to Early Tenth Centuries BCE. In Honor of Professor Trude Dothan, Jerusalem 1998.

Hnila, P. - Pieniazek-Sikora, M., Troia in the Early Iron Ages (Troia VIIb1-3), in: Troy. Journey to a City Between Legend and Reality, Istanbul 2002, 94-103.

Hochstätter, A., Kastanas. Die handgemachte Keramik, Berlin 1984.

Hoffner, H. A., The Last Days of Khattusha, in: Ward/Joukowsky (1992), 46-52.

Klengel, H., „Hungerjahre“ in Hatti, AoF 1 [1974], 165-174.

Koppenhöfer, D., Troia VII-Versuch einer Zusammenschau einschließlich der Ergebnisse des Jahres 1995. Mit einem Beitrag von Ralf Becks, StTroica 7 [1997], 295-353.

Koppenhöfer, D., Buckelkeramik und Barbarische Ware in Troia: Anmerkungen zur Herkunft, in: Aslan, R. - Blum, S. - Kastl, G. - Schweizer, F. - Thumm, D. (Hrsg.), Mauerschau. Festschrift für Manfred Korfmann, Remshalden-Grunbach 2002, 679-704.

${ }_{30} \mathrm{Zu}$ Migrationsbewegungen grundlegend Yakar (2003), 16f.; siehe auch Yakar (1993), $3 \mathrm{ff}$.

31 Jüngst an einschlägiger Keramik aus Troia VIIb vorgenommene Tonanalysen brachten das erstaunliche Ergebnis, daß deren chemischer „Fingerabdruck“ mit dem balkanischer Tonlagerstätten übereinstimmt. Dies läßt sich nur damit erklären, daß die untersuchten Gefäße nicht in Troia hergestellt worden sein können, sondern aus Südosteuropa stammen müssen (siehe Pinążek-Sikora (2002), 706 mit Anm. 15), es sei denn, die Tone selbst wären importiert worden, was jedoch wenig wahrscheinlich ist. 
Menghin, W. (Hrsg.), Schliemann und Troia, München 1992.

Muscarella, O. W., The Iron Age Background of the Phrygian State, BASOR 299/300 [1995] 91-101.

Niemeier, W.-D., The Mycenaeans in Western Anatolia and the Problem of the Origin of the Sea Peoples, in: Gitin u. a. (1998), 17-65.

Omura, S., 1990 Yılı Kaman-Kalehöyük Kazıları. 13. Kazı Sonuçları Toplantısı, Ankara 1991, 319-336.

Orthmann, W., Die Keramik der frühen Bronzezeit aus Inneranatolien (IstForsch. 24), Berlin 1963.

Ökse, T., Yukarı Kızılırmak Havzası Tunç Çağları ve Demirçağ Yerleşim Tarihi. Siedlungsgeschichte des oberen Kızllırmak-Gebietes von der Frühbronze- bis zur Eisenzeit. Belleten 62 [1999] 299-390.

Özdoğan, M., Taşlıcabayır. A Late Bronze Age burial mound in Eastern Thrace. Anatolica 14 [1987] 5-39.

Özdoğan, M., The Second Millennium of the Marmara Region. The Perspective of a Prehistorian on a Controversial Historical issue. IstMitt. 43 [1993] 151-163.

Pinążek-Sikora, M., Neue Anregungen zur Diskussion über die Beziehungen zwischen Troia und dem nordwestpontischen Gebiet, in: Aslan R. - Blum, S. - Kastl, G. - Schweizer, F. - Thumm, D. (Hrsg.), Mauerschau, Festschrift für Manfred Korfmann, Remshalden-Grunbach 2002, 705-715.

Postgate, N., Identifying the End of the Hittite Empire. Problems of reuniting history and archaeology at Kilise Tepe. Bilkent University Newsletter of the Department of Archaeology and History of Art 4 [2005] 26-30.

Sams, G. K., Observations on Western Anatolia, in: Ward/Joukowsky (1992), 56-60.

Seeher, J., Die Zerstörung der Stadt Hattuša, in: Wilhelm, G. (Hrsg.) Akten des IV. Internationalen Kongresses für Hethitologie. Studien zu den Boğazköy-Texten 45, Wiesbaden 2001, 623-634.

Silberman, N.A., The Sea Peoples, the Victorians and Us: Modern Social Ideology and Changing Archaeological Interpretations of the Late Bronze Age Collapse, in: Gitin u. a. (1998), 268-275.

Strobel, K., Aspekte eines neuen Bildes der Entwicklung Anatoliens in der Frühen Eisenzeit (a new understanding of the historical development of Anatolia in the Early Iron Age: the myth of the ,Dark Ages'), in: Çilingiroğlu, A./Darbyshire, G. (Hrsg.), Anatolian Iron Ages 5. Proceedings of the Fifth Anatolian Iron Ages Colloquium held at Van, 6-10 August 2001, Ankara/London 2005, 195-210.

Sürenhagen, D., Politischer Niedergang und kulturelles Nachleben des hethitischen Großreiches im Lichte neuerer Forschung, in: Magen, U. - Rashad, M. (Hrsg.), Vom Halys zum Euphrat. Thomas Beran zu Ehren, Münster 1996, 283-293.

Tezcan, B., 1964 Koçumbeli Kazısı: Koçumbeli Excavation 1964, Ankara 1966.

Toker, A. - Öztürk, J. (Hrsg), Museum of Anatolian Civilizations Metal Vessels, Ankara 1992.

Tolstikov, W. P. - /Trejster, M. J., Der Schatz aus Troja. Schliemann und der Mythos des Priamos-Goldes, Stuttgart/Zürich 1996.

Voigt, M. M. - Henrickson, R. C., The Early Iron Age at Gordion: The Evidence from the Yassihöyük Stratigraphic Sequence, in: Oren, E. D. (Hrsg.), The Sea Peoples and Their World: A Reassessment, Philadelphia 2000, 327-360.

Ward, W. A. - Joukowsky, M.S. (Hrsg.), The Crisis Years: The $12^{\text {th }}$ Century B.C. From Beyond the Danube to the Tigris, Dubuque 1992.

Yakar, J., Anatolian Civilization following the disintegration of the Hittite Empire: An Archaeological Appraisal. Tel Aviv 20 [1993] 3-28.

Yakar, J., Identifying Migrations in the Archaeological Records of Anatolia, in: Fischer u. a. (2003), 11-19.

Zimmermann, T., Die bronze- und früheisenzeitlichen Troiafunde der Sammlung Heinrich Schliemann im Römisch-Germanischen Zentralmuseum. Kataloge des Römisch-Germanischen Zentralmuseums, Mainz [im Druck].

Dr. Thomas Zimmermann M. A.

Bilkent University

Faculty of Humanities and Letters

Department of Archaeology and History of Art

TR - 06800 Bilkent, Ankara

zimmer@bilkent.edu.tr 

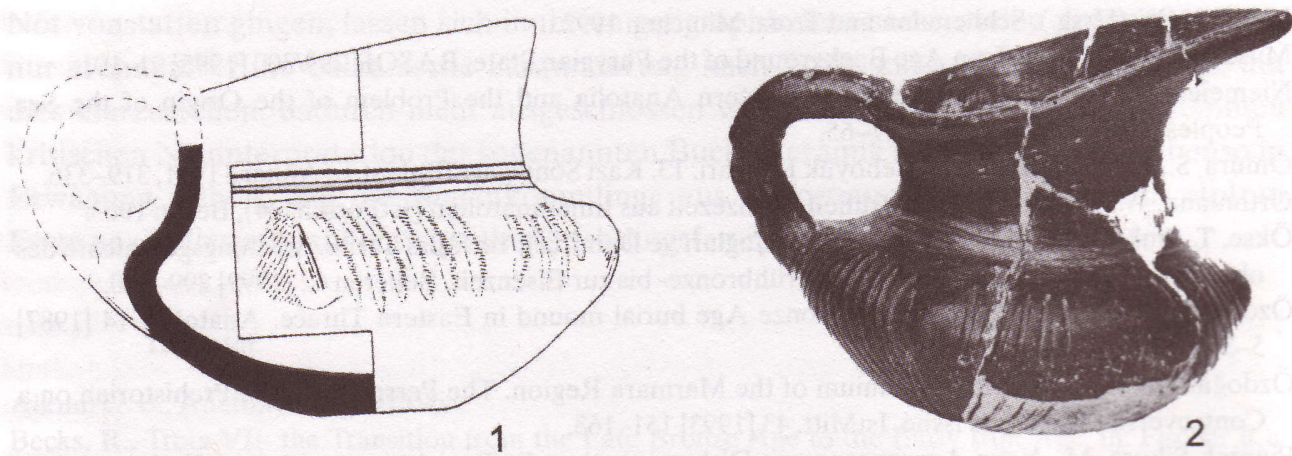

Abb. 1 Früheisenzeitliche Buckeltasse aus Troia Schicht VIIb2 (1) und frühbronzezeitliche Tasse mit Buckel- und Riefenzier aus Karaoğlan (2). Nach Zimmermann (1) und Alkım (1968) (2). Ohne Maßstab.

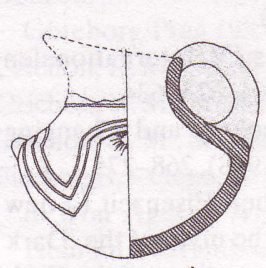

1

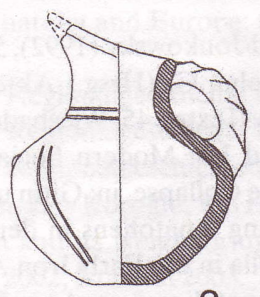

2

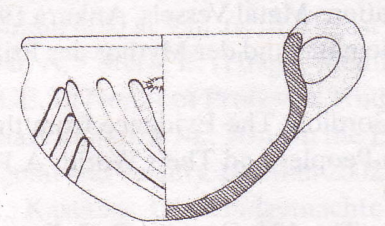

5

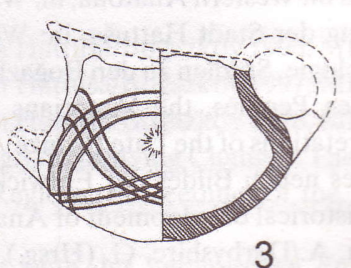

3

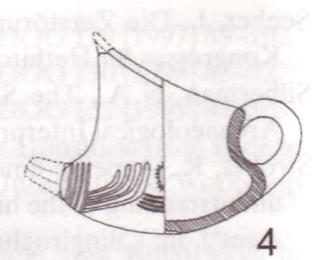

Abb. 2 Beispiele frühbronzezeitlicher buckel- und riefenverzierter Keramik aus Ahlatlıbel (1-4) und

Alaca Höyük (5-6). Nach Orthmann (1963). M=1:3.

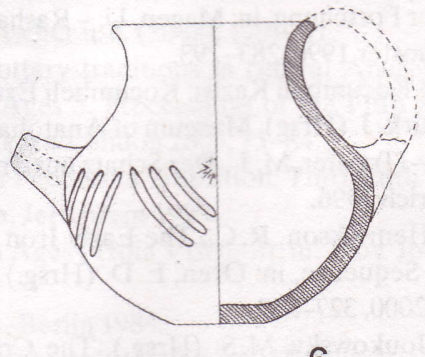

6 


$$
9
$$

